\title{
Contiguity and covariation in human causal inference
}

\author{
MARC J. BUEHNER \\ Cardiff University, Cardiff, Wales
}

\begin{abstract}
Nearly every theory of causal induction assumes that the existence and strength of causal relations needs to be inferred from observational data in the form of covariations. The last few decades have seen much controversy over exactly how covariations license causal conjectures. One consequence of this debate is that causal induction research has taken for granted that covariation information is readily available to reasoners. This perspective is reflected in typical experimental designs, which either employ covariation information in summary format or present participants with clearly marked discrete learning trials. I argue that such experimental designs oversimplify the problem of causal induction. Real-world contexts rarely are structured so neatly; rather, the decision about whether a cause and effect co-occurred on a given occasion constitutes a key element of the inductive process. This article will review how the event-parsing aspect of causal induction has been and could be addressed in associative learning and causal power theories.
\end{abstract}

Psychological research on human causal learning has largely adopted David Hume's (1739/1888) framework, according to which causality cannot be observed directly in the environment (see also Young, 1995, for an analysis of the Humean cues to causality). Although certain physical events may appear to give rise to instant causal perception (such as one billiard ball setting in motion a stationary ball by colliding with it), there is actually nothing in the event itself that can assure us of the causal relation (the ball could have been moved, for instance, by an ingenious magnetic mechanism from below). ${ }^{1}$ However, as Hume observed, two important principles can guide us in our search for causal explanations: the contingency between two events, and their contiguity. Starting with contiguity, the obvious constraint imposed by Hume is that causes need to be followed by their effects immediately in order to be credited with causality. ${ }^{2}$ But contiguity alone is of course not sufficient for causality, as two events could have followed each other merely by chance. Hence the importance of contingency or regularity: Only if two things repeatedly and reliably follow each other do we infer that they are causally related.

As this volume testifies, the vast majority of work on human causal learning, particularly in the last decade or so, has been exclusively concerned with the causal evaluation of contingency data (see Shanks, Holyoak, \& Medin, 1996, for an overview). One reason for this could be that a wide range of computational models have been prof-

I thank Jon May for helpful discussions on much of the work reviewed in this article, and for creating Figure 2. I also thank Lorraine Allan and Oskar Pineño for helpful comments on an earlier draft. Correspondence should be addressed to M. J. Buehner, School of Psychology, Cardiff University, P.O. Box 901, Cardiff CF10 3YG, Wales (e-mail: buehnerm@ cardiff.ac.uk). fered to explain how contingency information can be transformed into a measure of causal strength, and, as the literature shows, researchers in the field are still engaged in debates over which measure is the appropriate one (e.g., Buehner, Cheng, \& Clifford, 2003; Cheng, 1997; Lober \& Shanks, 2000; White, 2003). What unifies all these approaches is the assumption that reasoners evaluate information about the (joint) presence or absence of a cause $c$ and an effect $e$ toward a measure of causal strength. In its most simple form, such information can be represented in a $2 \times 2$ contingency table, as depicted in Figure 1.

A long-standing proposal (see, e.g., Allan \& Jenkins, 1980; Jenkins \& Ward, 1965) has been that causal strength is determined by subtracting the probability of the effect in the absence of the cause, $P(e \mid \neg c)=c /(c+d)$ from the probability of $e$ in the presence of $c, P(e \mid c)=a /(a+b)$. This measure $\Delta P=P(e \mid c)-P(e \mid \neg c)$ is commonly accepted as the mathematical formalization of contingency, and for many years has also been heralded as a normative measure of causation. At the same time, several studies have consistently reported that causal judgments often deviate systematically from $\Delta P$ (e.g., Wasserman, Elek, Chatlosh, \& Baker, 1993; see Cheng, 1997, for an overview), resulting in a plethora of suggestions - ranging from weighted probability contrasts or decision rules to associative learning networks - of how exactly the four entries in the contingency table are subjectively transformed into impressions of causal strength.

A consequence of the debate over what exactly is computed was that the question how it can be computed got somewhat lost along the way. In particular, a fundamental assumption underlying a contingency-based accountnamely, that reasoners have at their disposal information about the contingency between $c$ and $e$-has largely been 


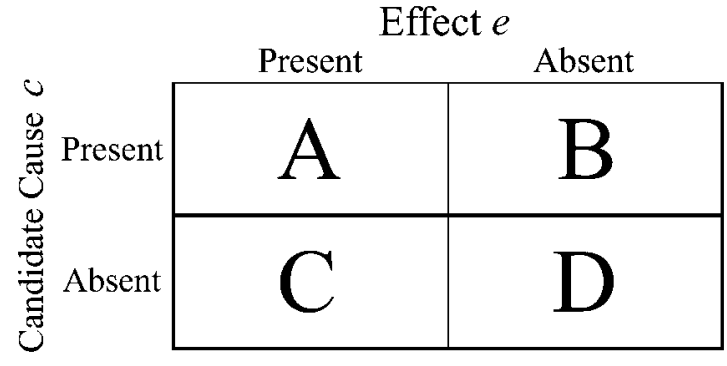

Figure 1. A standard $2 \times 2$ contingency table.

taken for granted. However, this represents an overly simplistic view of causal inference. Deciding whether or not $c$ and $e$ have co-occurred on a given occasion is an important precursor of causal inference. ${ }^{3}$ Consider, for example, a hypothetical experiment on instrumental learning where learning trials are defined by discrete time bins of a certain length $s$. As far as the experimenter is concerned, $c-e$ pairings that fall within a time bin contribute toward the A cell in the contingency table, whereas $c-e$ pairings that span a bin boundary are coded as one instance of cell B ( $c$, but no $e$ ), and a separate instance of cell $\mathrm{C}$ ( $e$, but no $c$ ). However, the participant most likely is completely unaware of this discrete trial structure, and therefore might adopt completely different standards of co-occurrence. If, for instance, a longer time frame of co-occurrence is assumed, event pairings that span a bin boundary might still be interpreted as instances of cell A. Subjectively perceived and objectively programmed contingency match only in the unlikely case of the participant adopting a subjective time frame that exactly matches the time bins implemented by the experi- menter. Even when programmed and subjective time frames match perfectly, there is still the possibility of pairings spanning a bin boundary. This is a serious limitation of the standard free-operant paradigm (see Buehner \& May, 2003, 2004, for detailed discussions). Figure 2 illustrates how a single sequence of events can be interpreted in multiple ways.

It is evident that any account of human causal learning is incomplete unless it addresses the determinants of (subjective) co-occurrence. The remainder of this paper will review empirical work relevant to this problem and will analyze the extent to which existing theories of causal learning can account for (or could be amended to account for) these results.

\section{Empirical Findings From Physical, Mechanistic, and Perceptual Causation}

Perhaps the earliest systematic investigations into the role of temporal contiguity and causal inference were carried out by Michotte (1946/1963) under the framework of perceptual causality. Michotte's participants observed a visual display of two stimuli A and B such that B was stationary while A moved toward it. In its classic form, the "launching display" then showed a collison of A with B, followed by B moving along the same trajectory as A, while A remained stationary after the impact with B. Participants reported compelling impressions of causality, such that A caused B to move, despite A and B being mere dots on a paper disk, rather than real physical objects. Michotte also found that inserting a delay of more than $100 \mathrm{msec}$ between impact and motion onset of $B$ destroyed the impression of immediate launching, and that delays of more than $150 \mathrm{msec}$ resulted in participants reporting two separate, independent movements.
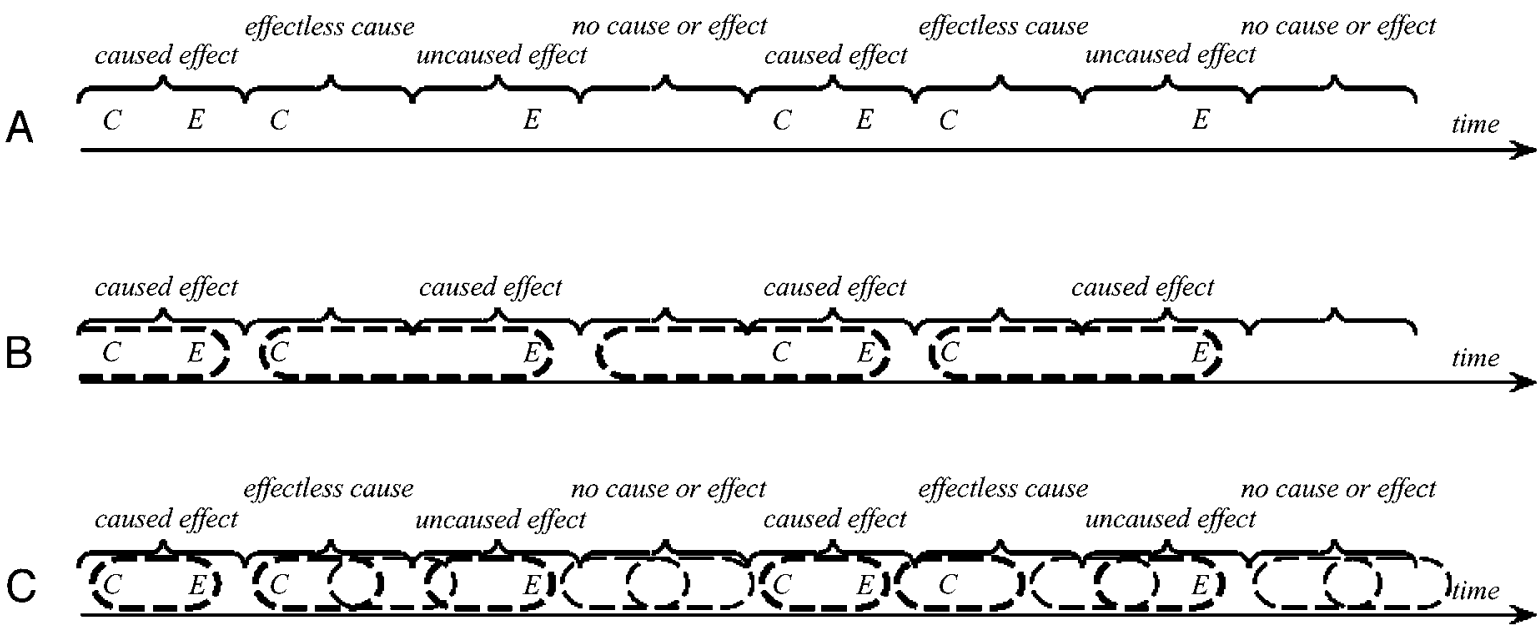

Figure 2. Parsing continuous event streams. The upper panel (A) shows two instances of each class of cause-effect pairing. Despite the underlying trial structure, every event follows a cause, albeit at varying intervals. An observer could parse the event stream as shown by the middle panel (B), where a hypothetical constant temporal window has been superimposed at each event. In the lower panel (C), a shorter temporal window provides the subjective impression of a cause-effect pairing as implemented by the trial structure, but there could be many more instances of windows containing neither cause nor effect, illustrated by the faint windows. 
The importance of temporal contiguity was also investigated extensively in a series of developmental studies. Siegler and Liebert (1974) presented 5- to 10-yearolds with the task of finding out what "made a light flash." There were two potential causes for the child to chose from: a discrete action performed by the experimenter (inserting a card into a computer), and a constant background cause (the computer exerting control over the light independently of the experimenter's actions). The experiment was set up in such a way that the action either always produced the effect, or on $50 \%$ of the trials. In addition, the temporal lag of the effect was either $0 \mathrm{sec}$ or $5 \mathrm{sec}$. Participants consistently rated immediate causes as stronger; more specifically, they preferred an immediate but imperfect predictor to a delayed but perfect predictor. Mendelson and Shultz (1976) presented their participants (4- to 7-year-olds) with a forced choice between a deterministic but delayed cause and a noncontingent but contiguous cause. The causes consisted of marbles being dropped in either one of two holes of a wooden box, and the effect consisted of a bell ringing inside a bell box. Participants consistently preferred the contiguous, noncontingent predictor. However, this trend was slightly modulated when a "model," consisting of a 34-in. rubber tube connecting the bottom of the wooden box with the bell box, was presented during learning.

The most important support for the centrality of contiguity in mechanical causation comes from Schlottmann (1999). Schlottmann exposed her participants (5- to 10year-olds, and adults) to a "mystery box" similar to the one used by Mendelson and Shultz (1976). Participants initially observed the experimenter drop one marble in hole $\mathrm{A}$, then a few seconds later drop another marble in hole $\mathrm{B}$, followed immediately by the bell ringing. All participants spontaneously attributed causality to the second, contiguous, Cause B. Participants then had a chance to explore the mystery box and learned that there were two different mechanisms that could be placed into the box and positioned under one of the two holes, so that balls dropped into that hole would set a mechanism in motion to make the bell ring. One mechanism was a "fast" see-saw, the other a "slow" runway. Participants could play with these mechanisms and learned about the respective delays associated with each mechanism. More specifically, participants of all age groups could successfully predict whether the bell would ring immediately or after a delay, depending on which mechanism was placed inside the box, and they could diagnose which mechanism was inside the box on the basis of the delay between dropping the ball and the bell ringing. The crucial test, however, was as follows. The experimenter placed one of the two mechanisms inside the box without telling participants under which of the two holes it had been placed. In order to make sure that young children remembered which mechanism was in the box, a representative sticker was attached to the outside of the box. The experimenter dropped one ball in hole A, waited a few seconds, dropped another ball into hole B, and then the bell rang immediately. Participants had to say which of the two balls had made the bell ring. Children up to 7 years consistently chose the second, contiguous, ball, even when they knew that the slow mechanism was inside the box. Older children and adults correctly chose the first, noncontiguous, ball on trials when the slow mechanism was inside the box, and the second, contiguous, ball when the fast mechanism was inside the box. Schlottmann's results show that the role of contiguity in causal learning is not to enable causal learning, for even the young children could successfully predict a long delay when the slow toy was in the box, and could correctly identify which of the two toys was in the box on the basis of the extent of the temporal delay. Rather, the role of contiguity seems to be more complex. According to Schlottmann, contiguity is a very strong cue for causality, but it is not the only one. The critical test in Schlottmann's study pitched contiguity against understanding of mechanism, and her results show that for young children, experienced contiguity overrides explicit knowledge of a slow mechanism; older children and adults, on the other hand, could successfully integrate information about contiguity and mechanism to come to a correct causal assessment.

\section{Empirical Findings From \\ Associative/Instrumental Learning}

The studies described in the preceding section were somewhat limited in that they nearly always employed deterministic causal relations. Much of our everyday causal learning concerns probabilistic relations, and this section will review how variations in contiguity affect causal inference from probabilistic data. A straightforward investigation was carried out by Shanks, Pearson, and Dickinson (1989), who exposed participants to an instrumental learning paradigm, requiring them to learn how strongly pressing a key produced illumination of a triangle displayed on the computer screen. The schedule was programmed not to produce the effect unless a response was emitted, $P(e \mid \neg c)=0$, and to produce outcomes with $P(e \mid c)=.75$ when a response was made. Participants were remarkably accurate at estimating the contingency correctly. However, when response and outcome were separated by $4 \mathrm{sec}$ or more, participants no longer could distinguish between causal and yoked control (i.e., noncontingent) conditions.

An interesting variation of Shanks et al.'s (1989) paradigm was implemented by Reed (1992, 1996, 1999), who amended the design described above to include a signaling procedure. The method was almost identical to that described in Shanks et al. (1989), except that in the delayed conditions a signal stimulus (a row of Xs) was displayed immediately after the keypress. Even though the signals were not mentioned in the instructions, Reed found that they significantly improved assessment of delayed contingencies. One could of course argue that the addition of the signal effectively removed the delay in these conditions: After all, participants in Reed's experiments received immediate feedback (in the form of a row of Xs) indicating that their action had been recorded 
by the computer and had had some effect. Indeed, Reed found that the effectiveness of the signal was dependent both on its contiguity with the response (i.e., a delayed signal halfway between response and outcome was less effective) and its contingency with the response.

Anderson and Sheu (1995, Experiment 2), in a study aimed at investigating the parametrization of the $\Delta P$ rule, noted that their participants frequently reported that they paid attention to the perceptual quality of the feedback (and how this quality changed as a function of the instrumental response) rather than the allocation of specific events to one of the four cells in the contingency table. In their Experiment 2, participants had to learn the relationship between clicking a button on the screen and the delivery of an auditory and a visual stimulus. Three values of $P(e \mid c)$ and $P(e \mid \neg c)$ were combined to produce nine different conditions. Depending on the specific condition, these probabilities were defined relative to 750 - or $1,500-\mathrm{msec}$ time bins (i.e., learning trials), and the outcome, if scheduled, was always presented at the end of each trial. Although a weighted $\Delta P$ model could fit the data to account for $97 \%$ of the variance, Anderson and Sheu conjectured that, at least in a continuous instrumental paradigm, causal learning might not actually be driven by the conditional probabilities $P(e \mid c)$ and $P(e \mid \neg c)$, but instead might be a function of outcome rates, conditional on the presence and absence of $c$, $\mathrm{R}(e \mid c)$ and $\mathrm{R}(e \mid \neg c)$. Since rates and probabilities were confounded in their experiment, they attempted to decorrelate them in their subsequent Experiment 3. In this experiment, a trial length $\mathrm{T}$ could take one of three values $(450,750$, or $1,050 \mathrm{msec})$; importantly, $\mathrm{T}$ was allowed to differ between trials when a response was emitted (TR) and trials on which no response was emitted $(T \neg R)$. There were three timing conditions: TR $(450 \mathrm{msec})<$ $\mathrm{T} \neg \mathrm{R}(1,050 \mathrm{msec}), \mathrm{TR}=\mathrm{T} \neg \mathrm{R}(750 \mathrm{msec})$, and $\mathrm{TR}$ $(1,050 \mathrm{msec})>\mathrm{T} \neg \mathrm{R}(450 \mathrm{msec})$. Results showed main effects of contingency and timing condition, as well as an interaction. In addition to being receptive to contingency, participants also were sensitive to contiguity: Causal ratings decreased as a function of TR. This sensitivity to contiguity persisted even when $P(e \mid c)=$ $P(e \mid \neg c)=1$ (Experiment 4). In this experiment, the outcome occurred at the end of every trial. However, depending on the timing condition, responding could either advance or postpone the delivery of a certain outcome (and, given a finite sampling interval, thus lead to more or less outcomes overall; see Wasserman \& Neunaber, 1986, for a similar result). Anderson and Sheu developed a grating contrast model based on relative rates and found that this model fitted the data better than the $\Delta P$ rule or a rate-based version of it $(\Delta R)$.

\section{Theoretical Accounts of the Role of Contiguity in Causal Learning}

The preceding sections have shown that contiguity plays a fundamental role in human causal learning from contingency data, in two ways. Contiguity seems to strongly fa- cilitate the discovery of causal relations, so that delayed relations are not detected at all (as in Shanks et al., 1989; or Michotte, 1946/1963), are underestimated (Shanks et al., 1989), or require additional resources (information integration, Schlottmann, 1999; or external prompting, Mendelson \& Shultz, 1976) to be assessed adequately. On the other hand, contiguity alone can sometimes be a sufficiently strong cue to elicit impressions of causality, even when contingency is comparatively low (Siegler \& Liebert, 1974) or even absent (Anderson \& Sheu, 1995; Mendelson \& Shultz, 1976; Wasserman \& Neunaber, 1986).

From a conceptual or computational perspective, explaining the facilitative role of contiguity is straightforward. If we interpret causal learning as a task that involves extracting regularities between putative causes and effects from a continuous flux of events, then contiguous pairings are obviously advantaged, for they are more likely to be discovered (if we assume the learner has limited resources of memory and attention). Delayed pairings are also more liable to attract intervening events, which in turn would need to be considered as potential competing causes, thus increasing the complexity of the reasoning problem.

The apparent sufficiency of contiguity found in instrumental paradigms, on the other hand, is less compelling. However, as Wasserman and Neunaber (1986) have noted, there are multiple ways in which participants could interpret the request to provide a rating of the effectiveness of the action with respect to the outcome. Ratings could reflect how causal actions change the overall rate (and thus quantity) of the outcome, the time of delivery, or the probability (specific to a certain time bin). In both Wasserman and Neunaber (1986) and Anderson and Sheu's (1995) experiments, actions clearly had a noticeable effect: They immediately produced an outcome that would otherwise have occurred later in time (or postponed an outcome that would otherwise have occurred at a set time). Although the action might not make a difference for the global amount of outcomes, it affects the local distribution pattern, and participants most likely are sensitive to both global and local patterns of occurrence.

In the following sections, I will review various theoretical accounts of the facilitative role of contiguity, and then evaluate the degree to which these accounts can explain the pattern of results.

\section{The Role of Temporal Contiguity in Associative Learning}

Most associationist researchers interested in human causal learning refer to the Rescorla-Wagner model (Rescorla \& Wagner, 1972) as a plausible algorithmic description of causal learning, although the Pearce (1987) model has recently attracted more attention, due to its capacity to account (to some extent) for base-rate effects that the Rescorla-Wagner model fails to explain (see, e.g., Buehner et al., 2003; Collins \& Shanks, 2003; Lober \& Shanks, 2000; Perales \& Shanks, 2003). Associationism 
lays strong claims to Hume's (1739/1888) empiricism and thus recognizes both contingency and contiguity as fundamental cornerstones for establishing associations. It is somewhat surprising then that "contemporary accounts are usually silent about the actual inter-event interval over which an association can be formed, but all argue that the size of the increment in associative strength accruing from a pairing decreases as the contiguity is degraded" (Shanks \& Dickinson, 1987, p. 231).

One reason why a formal representation of the role of contiguity might not have seemed necessary for the authors of these models could be that they interpreted contiguity as restricted to its facilitative role (Allan, Tangen, Wood, \& Shah, 2003). While contiguity promotes the growth and thus the strength of the cause-effect association, no information about it is acquired during the learning process: "Experience is stored as a small number of associative strengths . . . information about past events is lost in the computation. In other words, these models do not have episodic memory" (Baker, Murphy, \& Vallée-Tourangeau, 1996, p. 1). As a consequence, poor contiguity is expected to slow down learning, but deficits in contiguity presumably could be alleviated (at least to a certain extent) by increasing the number of learning trials; a small number of contiguous trials would be predicted to elicit results similar to those elicited by a large number of delayed trials.

Not all associative theories adopt such a restricted view of contiguity, however. According to the temporal coding hypothesis (Miller \& Barnet, 1993; Savastano \& Miller, 1998), for instance, temporal information is encoded alongside the association. Rather than merely shaping how quickly an association is learned, the degree of contiguity also determines the quality of what is learned. More specifically, the difference between being exposed to a delayed or a contiguous contingency would not only be that the former might elicit weaker judgments of causal strength than the latter; in addition, each would create concomitant expectations of delay or contiguity, so that presentation of the cause (subsequent to learning) produces anticipation of the effect after the same time interval as that encountered during learning.

\section{The Role of Temporal Contiguity in \\ Knowledge-Based and Probabilistic Theories}

Knowledge-based theories interpret causal learning as a process consisting of an interaction between bottomup (contingency learning) and top-down (knowledgemediation) processes (Waldmann, 1996). More specifically, prior knowledge determines how the evidence at hand is parsed (Hagmayer \& Waldmann, 2002) and evaluated (Waldmann \& Hagmayer, 1999; Waldmann \& Holyoak, 1992). A formal account of the role of temporal contiguity has been offered by Einhorn and Hogarth (1986). They stated that contiguity privileges contingencies to be interpreted as causal. However, in the absence of contiguity, relations could still be evaluated as causal if the reasoner were aware of some causal chain or mech- anism that makes the delay plausible and thus bridges the temporal gap. In the absence of such knowledge, delayed contingencies would be rated as noncausal. Crucially, this knowledge-mediation hypothesis also entails a second postulation: If the reasoner assumes that the causal mechanism at work necessitates a delay (e.g., the relation between smoking and lung cancer), immediate pairings should not be credited with causality but should instead be deemed spurious (e.g., a diagnosis of lung cancer 1 day after one has taken up smoking cannot be related to smoking). The role of contiguity in knowledge-based theories thus is more complex than in associationist theories in that the lack of contiguity need not necessarily always lead to poorer causal learning. Depending on the prior knowledge, lack of contiguity might be inconsequential or even beneficial for learning.

Recent knowledge-based theories mostly adopt a probabilistic notion of causality (e.g., Waldmann, 1996), and a logical question to ask is how the top-down knowledge components interact with the bottom-up probability learning elements to explain the complex role of contiguity in causal learning. The function of contiguity is that it modulates the interpretation of a given cause-effect pairing as instances of cell A, B, C, or D in the contingency table. In the absence of any knowledge (i.e., when contiguous relations are favored), a contiguous pairing thus might count as an instance of cell A, but a delayed pairing might count as one instance of $B$ and a separate instance of $\mathrm{C}$. This same delayed pairing might be evaluated as an instance of A, if the reasoner assumes a delayed causal relation. In other words, the function of delays might be to shift causal attribution away from the candidate cause toward a background of alternative causes, thus decreasing $\Delta P$ by simultaneously decreasing $P(e \mid c)$ and increasing $P(e \mid \neg c)$, with the presence and direction of the attribution shift being determined by knowledge or assumptions about the causal mechanism in question.

\section{The Role of Temporal Contiguity in Rate-Based Accounts}

The essence of the associationist framework is to provide a parsimonious account of human causal learning by mapping theories and results from animal learning to human learning. However, Gallistel and Gibbon (2000) have recently proposed an alternative account to explain a wide range of animal conditioning phenomena. Their rate-estimation theory (RET) radically breaks with the traditions of associationism in that it challenges the notion that conditioning is governed by associative laws. According to Gallistel and Gibbon, temporal information, rather than association, is the crucial force behind conditioning. Whereas the temporal coding hypothesis (Miller \& Barnet, 1993; Savastano \& Miller, 1998) assumes that temporal information is encoded in addition to associative strength, RET postulates that temporal information is all that is acquired. In RET, organisms keep track of the rate of outcome occurrence and how this rate changes as a function of the presence of the cause or cue. 
Whether and when responding occurs (and when it stops) is governed by a decision process involving a ratio of the outcome rates. Contiguity thus is central to this account since it determines whether a specific outcome contributes toward the rate conditional on the presence or absence of the cause. Interestingly, however, the common finding that degraded contiguity leads to weaker responses is attributed to a methodological artifact according to RET: Usually, delay or trace conditioning procedures involve manipulations of the cue-outcome interval only. If this interval is increased, then the rate ratio changes, and acquisition is retarded (as accounted for by associative theories). However, if the intertrial interval is adjusted proportionally to the cue-outcome interval (and the organism is exposed to longer time periods where neither cue nor outcome is present), the rate ratio remains unchanged, and delays no longer impair learning. This property is known as time-scale invariance.

Related to RET is relative contiguity (Wasserman \& Neunaber, 1986), which likewise postulates that the organism is sensitive to changes in rate of outcome occurrence, conditional on the presence of a cue of cause. Such rate-based accounts work well to account for Anderson and Sheu's (1995) or Wasserman and Neunaber's results showing that causal ratings are sensitive to causes merely advancing the outcome in time (or changing relative rates), without affecting the probability of occurrence. They are compromised, however, by the fact that they cannot really address point events. In animal learning, the stimuli are always presented for extended periods of time, and these time periods serve as the basis for calculating the rate of outcome occurrence in the presence or absence of the cue. Most human causal learning tasks, however, involve point events of extremely short duration (e.g., pressing a key). It is thus impossible to calculate an outcome rate conditional on the presence of the cause, unless one hypothesizes a time window of causal activity. This is of course possible, but the size of the temporal window would presumably be governed by prior knowledge, rendering the rate-based account remarkably similar to the knowledge-based account, with the only difference being that one would track rates and the other probabilities. The advantage of the rate-based account, of course, is that rates, unlike probabilities, do not have an upper limit of 1 .

\section{Evaluation of Associative Versus Knowledge-Based Accounts}

The review of relevant data from mechanistic and associative studies of causal learning showed that the typical finding in the literature has been that cause-effect delays impair causal learning. An associationist account can explain this globally by pointing at the facilitative role contiguity plays in acquiring associations. The knowledgemediation account, on the other hand, would consider each specific study to find out what the expected time frame of the causal relation in question most likely would have been. In Michotte's (1946/1963) launching para- digm, for instance, the display created the illusion of physical objects in motion. When evaluating such a situation, participants bring to the task their naive understanding of the properties of physical impact (e.g., if mass and velocity are sufficiently large, impact of one object with another will result in immediate motion of the object suffering impact). A display of a delayed launch violates these expectations and is thus seen as noncausal. Such a mismatch between knowledge/expectation and experience can also explain the detrimental effect of delay in Shanks et al.'s (1989) studies: Because the experiment was carried out on a computer, participants would have applied their knowledge about computers (including that actions on computers usually have immediate consequences) to the task. When this assumption of immediacy was violated, the relation was not interpreted as causal (the same explanation would apply for Siegler \& Liebert, 1974).

Recent work (Buehner \& May, 2002, 2003, 2004) disentangles predictions derived from associative and knowledge-based accounts by manipulating participants' knowledge of the time frame of the causal relation. One study involved implicit manipulations (Buehner \& May, 2002) and presented participants with two different causal mechanisms to induce assumptions of immediacy (switching on a lightbulb) or delay (firing off a grenade and observing an explosion). Other studies involved explicit instructions about potential delays (Buehner \& May, 2003, 2004). Across all studies, knowledge of potential delays significantly improved the assessment of delayed relations, and in one case (Buehner \& May, 2004) even removed the detrimental influence of delay completely.

These results are at variance with most associationist accounts, since interpretation of the very same stimuli varied as a function of higher level knowledge, which such accounts fail to represent. While the toleration of delays from participants who were instructed about temporal gaps certainly fits with a knowledge-based account, these results could not provide full-fledged support for it. According to this account, immediate pairings should be disregarded as spurious, if the reasoner assumes a delayed relation. However, the above-mentioned studies consistently found that immediate relations were always rated as highly causal, even when participants were instructed to expect delays. This finding is most likely an artifact of the instrumental procedure employed: Irrespective of the instructions, every causal action performed by the participant produced the effect according to the programmed probability. It was thus only rational for participants to report that their actions produced the effect, even when they expected a delay, particularly if one takes into account that they interacted with a computer and knew that delivery of outcomes occurred according to an underlying program, and that the cover story was essentially made up.

Full-fledged support for a knowledge-based account came from Allan et al. (2003), who replicated Buehner and May's (2002, Experiment 3) design in an observational learning paradigm. Participants watched a series 
of learning trials depicting a military officer either pressing a fire button $(c)$ or standing still $(\neg c)$, followed by a depiction of an explosion $(e)$, or nothing $(\neg e)$. Explosions were displayed after a delay of $0 \mathrm{sec}$ or $5 \mathrm{sec}$. Participants received instructions stating either that the fire button operated a radio-controlled detonator to set off explosions in a military training range (immediate expectation), or that it launched off a grenade that would travel into range and explode there (delay expectation). Allan et al. replicated our results, but additionally found that immediate contingencies were rejected as noncausal when participants expected a delay.

Allan et al. (2003) also pointed out that these results can likewise be accommodated for by the temporal coding hypothesis (Miller \& Barnet, 1993; Savastano \& Miller, 1998). In order to explain knowledge-mediation effects using the temporal coding hypothesis, one would have to assume that the instructions serve as a preliminary learning phase during which the temporal relation between cause and effect is encoded. The contingency experienced in the subsequent "real" learning phase will attract higher ratings if its time frame is congruent to the one encoded in the preliminary phase (because it fits with the expectations generated in the preliminary phase). It is not clear, however, how this interpretation of the temporal coding hypothesis differs from the original knowledge-mediation account.

Full support for the knowledge-mediation account also comes from Hagmayer and Waldmann (2002), who investigated how participants aggregated covariational data from unstructured lists. Participants had to study longitudinal data tabulating whether or not a mosquito plague had affected a hypothetical community, and whether or not an anti-mosquito intervention had taken place. The lists represented a time period of 20 years, with entries about the presence and absence of the effect (absence of mosquito plague) and the cause (intervention) listed for every year. Ingeniously, the data was structured in such a way that the cause-effect contingency was slightly negative $(-.125)$ when assessed within the same year, but moderately positive $(+.292)$ if assessed from one to the next year. In addition, participants were presented with two different cover stories. One stated that the intervention consisted of application of an insecticide, whereas the intervention in the other story was the planting of special plants that allow mosquito-larvae-eating beetles to breed. Although time frame was not mentioned explicitly, Hagmayer and Waldmann assumed that the former would elicit expectations of immediacy, whereas the latter would elicit expectations of delay. As predicted by a knowledge-based account, the scope of contingency aggregation was determined by the instructions. Participants evaluated the very same data set as either supporting a causal relation (if they had received the plant cover story) or rejecting this relation (if they had received the insecticide story).

As explained earlier, associative and probabilistic theories differ in their postulated function of cause-effect delays. According to the former, delays result in weaker increments of associative strength on any given trial, whereas according to the latter, the function is to shift attribution away from $P(e \mid c)$ to $P(e \mid \neg c)$. Each prediction has been tested in turn. The attribution-shift hypothesis was first raised by Shanks and Dickinson (1987). In order to test it, they exposed participants to an instrumental task involving two response keys at a time. In a control condition, both keys A1 and A2 produced the outcome with $P(e \mid c)=.75$, and $P(e \mid \neg c)$ was .25 (relative to some unspecified time bin, presumably $1 \mathrm{sec}$ ), and there was no cause-effect delay. The experimental condition involved keys A3 and A4, which had the same probabilities attached to them as A1 and A2; crucially, however, pressing A4 produced the outcome after a 4 -sec delay, while A3 was linked with a 0 -sec delay. In line with earlier results, Shanks and Dickinson found that causal ratings for $\mathrm{A} 1$ and $\mathrm{A} 2$ were remarkably accurate, and that participants underestimated the contingency associated with A4. The crucial test for the attributionshift hypothesis, however, concerned judgments for A3. A3 received judgments that were higher than either the objective contingency or the ratings of Control Conditions A1 and A2. Shanks and Dickinson interpreted this as evidence against the attribution-shift hypothesis:

If the effect of contiguity is mediated by a change in the perceived contingency, we should expect to have observed a decrement in the judgments not only for A4 but also for A3. According to this account, delaying the outcome for A4 does not just decrease the perceived $P(\mathrm{O} \mid$ A4 $)$ but also correspondingly increases $P(\mathrm{O} \mid \neg \mathrm{A} 4)$. As these delayed outcomes were unlikely to have occurred in close association with A3, they should also have served to increment $P(\mathrm{O} \mid \neg \mathrm{A} 3)$, thus reducing the perceived contingency for A3 as well as A4. (p. 235)

Because Shanks and Dickinson's (1987) experiment contained three potential causes (A3, A4, and a constant background of alternative causes), an attribution shift away from A4 could benefit attribution toward A3, the constant background, or a combination of both. Without a detailed analysis of the response-outcome patterns generated by participants, it is impossible to fully test the predictions of the attribution-shift hypothesis. Shanks and Dickinson's report did not contain such analyses, so their interpretation at best is speculative. However, the fact that $\mathrm{A} 3$ attracted ratings higher than both the objective $\Delta P$ and the control conditions suggests that some of the delayed outcomes produced by A4 might have in fact been attributed to A3, contrary to Shanks and Dickinson's speculation, but as predicted by the attributionshift hypothesis.

\section{Avoiding Circularity: Where Does the Knowledge Come From?}

Knowledge-based theories of causation are often criticized for their inherent circularity: In order to evaluate a causal relation as causal, one first needs to know that there is a causal mechanism (see Cheng, 1997). Through- 
out this paper I have consistently shown that the impact of temporal contiguity on causal learning (and thus the determinants of subjective co-occurrence) is mediated by the reasoner's prior knowledge. However, all prior knowledge (at least of the kind considered here) is of course ultimately grounded in experience. The question thus is how such knowledge might be acquired without initial topdown support. In many instances, such knowledge could have been acquired via cultural transmission, through analogies, or through understanding of physical constraints, not necessarily tied to causal learning. However, there are also numerous situations where these options are not available, and causal learning of delayed relations still takes place, presumably by some boot-strapping process. In other words, there must be processes that allow us to extract hypotheses about causal relations (and the potential time frames associated with them) from unstructured continuous evidence. My colleagues and I are currently investigating a number of ways that such processes could operate, and it is clear that many questions still remain unanswered (and perhaps even unasked).

Overall, however, the evidence so far suggests that a contingency-based account of causal judgment is well justified, as long as it recognizes the need for an eventparsing algorithm that determines how individual event pairings are interpreted in terms of allocating them to the four cells $\mathrm{A}, \mathrm{B}, \mathrm{C}$, and $\mathrm{D}$. The output of this parsing algorithm directly feeds into the causal learning algorithm. It is evident that none of the causal learning algorithms discussed in this paper can account for the rich pattern of findings related to temporal structure, although some (relative contiguity, RET, temporal coding hypothesis) at least attempt to address temporal structure in their representations, while others (probabilistic accounts, certain variants of associationism) completely fail to address representations of cause-effect timing. Failure to address temporal structure does not necessarily discredit these approaches altogether, as long as they can be amended to allow external event-parsing algorithms to carve the flux of events into meaningful units to serve as inputs for their computations. The approaches that do address temporal structure are entirely knowledge lean and thus cannot account for the knowledge-mediation effects reviewed in this paper. One could of course add knowledge-mediation into rate-based accounts, but it is not clear how this would differ from a probabilistic account where the time frame of probability is determined by prior knowledge.

Because many of the event-parsing effects reviewed involved a mediation of the role of temporal structure by higher level knowledge, it is most parsimonious to assume that the causal learning algorithm is one that is likewise sensitive to higher level knowledge. Waldmann and colleagues have shown that higher level knowledge influences both the parsing and aggregation of data over time (Hagmayer \& Waldmann, 2002), and the interpretation of contingency data, once it has been parsed (Waldmann, 2000, 2001; Waldmann \& Holyoak, 1992), thus overall strengthening the case for causal induction as a knowledge-based process (Waldmann, 1996).

\section{REFERENCES}

Allan, L. G., \& Jenkins, H. M. (1980). The judgment of contingency and the nature of response alternatives. Canadian Journal of Psychology, 34, 1-11.

Allan, L. G., TANGen, J. M., Wood, R., \& Shah, T. (2003). Temporal contiguity and contingency judgments: A Pavlovian analogue. Integrative Physiological \& Behavior Science, 31, 205-211.

Anderson, J. R., \& Sheu, C. F. (1995). Causal inferences as perceptual judgments. Memory \& Cognition, 23, 510-524.

Baker, A. G., Murphy, R. A., \& Vallée-Tourangeau, F. (1996). Associative and normative models of causal induction: Reacting to versus understanding cause. In D. R. Shanks, K. J. Holyoak, \& D. L. Medin (Eds.), The psychology of learning and motivation: Vol. 34. Causal learning (pp. 1-45). San Diego: Academic Press.

Buehner, M. J., Cheng, P. W., \& Clifford, D. (2003). From covariation to causation: A test of the assumption of causal power. Journal of Experimental Psychology: Learning, Memory, \& Cognition, 29, $1119-1140$

BUEHNER, M. J., \& MAY, J. (2002). Knowledge mediates the timeframe of covariation assessment in human causal induction. Thinking \& Reasoning, 8, 269-295.

BUEHNER, M. J., \& MAY, J. (2003). Rethinking temporal contiguity and the judgment of causality: Effects of prior knowledge, experience, and reinforcement procedure. Quarterly Journal of Experimental Psychology, 56A, 865-890.

BUEHNER, M. J., \& MAY, J. (2004). Abolishing the effect of reinforcement delay on human causal learning. Quarterly Journal of Experimental Psychology, 57B, 179-191.

CHENG, P. W. (1993). Separating causal laws from casual facts: Pressing the limits of statistical relevance. In D. L. Medin (Ed.), The psychology of learning and motivation: Vol. 30. Advances in research and theory (pp. 215-264). San Diego: Academic Press.

Cheng, P. W. (1997). From covariation to causation: A causal power theory. Psychological Review, 104, 367-405.

Collins, D., \& ShanKs, D. (2003). Conformity to the power PC theory depends on the type of probe question. Manuscript submitted for publication.

EInHoRn, H. J., \& Hogarth, R. M. (1986). Judging probable cause. Psychological Bulletin, 99, 3-19.

GaLlistel, C. R., \& GibBON, J. (2000). Time, rate, and conditioning. Psychological Review, 107, 289-344.

HAGMAYER, Y., \& WALDMANN, M. R. (2002). How temporal assumptions influence causal judgments. Memory \& Cognition, 30, 11281137.

Hammond, L. J., \& Paynter, W. E. (1983). Probabilistic contingency theories of animal conditioning: A critical analysis. Learning \& Motivation, 14, 527-550.

HumE, D. (1888). A treatise of human nature. In L. A. Selby-Bigge (Ed.), Hume's treatise of human nature. Oxford: Oxford University Press, Clarendon Press. (Original work published 1739)

JENKINS, H. M., \& WARD, W. C. (1965). Judgment of contingency between responses and outcomes. Psychological Monographs: General \& Applied, 79(1, Whole No. 594), 1-17.

LOBER, K., \& SHANKS, D. R. (2000). Is causal induction based on causal power? Critique of Cheng (1997). Psychological Review, 107, 195212.

Mendelson, R., \& Shultz, T. R. (1976). Covariation and temporal contiguity as principles of causal inference in young children. Journal of Experimental Child Psychology, 22, 408-412.

Michotтe, A. E. (1963). The perception of causality (T. R. Miles, Trans.). London: Methuen. (Original work published 1946)

Miller, R. R., \& BARNET, R. C. (1993). The role of time in elementary associations. Current Directions in Psychological Science, 2, 106111.

Pearce, J. M. (1987). A model for stimulus generalization in Pavlovian conditioning. Psychological Review, 94, 61-73.

Perales, J. C., \& ShANKS, D. R. (2003). Normative and descriptive accounts of the influence of power and contingency on causal judgement. Quarterly Journal of Experimental Psychology, 56. 977 1007.

REED, P. (1992). Effect of a signaled delay between an action and out- 
come on human judgment of causality. Quarterly Journal of Experimental Psychology, 44B, 81-100.

REED, P. (1996). No evidence for blocking in human judgments of causality by stimuli presented during an outcome delay. Learning \& Motivation, 27, 317-333.

REED, P. (1999). Role of a stimulus filling an action-outcome delay in human judgments of causal effectiveness. Journal of Experimental Psychology: Animal Behavior Processes, 25, 92-102.

REscorla, R. A., \& Wagner, A. R. (1972). A theory of Pavlovian conditioning: Variations in the effectiveness of reinforcement and nonreinforcement. In A. H. Black \& W. F. Prokasy (Eds.), Classical conditioning II: Current theory and research (pp. 64-99). New York: Appleton-Century-Crofts.

Savastano, H. I., \& Miller, R. R. (1998). Time as content in Pavlovian conditioning. Behavioural Processes, 44, 147-162.

Schlottmann, A. (1999). Seeing it happen and knowing how it works: How children understand the relation between perceptual causality and underlying mechanism. Developmental Psychology, 35, 303317.

Scholl, B. J., \& NaKayama, K. (2002). Causal capture: Contextual effects on the perception of collision events. Psychological Science, 13, 493-498.

ScHOLL, B. J., \& Tremoulet, P. D. (2000). Perceptual causality and animacy. Trends in Cognitive Sciences, 4, 299-309.

ShanKs, D. R., \& DiCKINSON, A. (1987). Associative accounts of causality judgment. In G. H. Bower (Ed.), Psychology of learning and motivation: Vol. 21. Advances in research and theory (pp. 229-261). San Diego: Academic Press.

Shanks, D. R., Holyoak, K. J., \& Medin, D. L. (EDS.) (1996). The psychology of learning and motivation. Vol. 34: Causal learning. San Diego: Academic Press.

Shanks, D. R., Pearson, S. M., \& Dickinson, A. (1989). Temporal contiguity and the judgment of causality by human subjects. Quarterly Journal of Experimental Psychology, 41B, 139-159.

Siegler, R. S., \& LieberT, R. M. (1974). Effects of contiguity, regularity, and age on children's causal inferences. Developmental Psychology, 10, 574-579.

Waldmann, M. R. (1996). Knowledge-based causal induction. In D. R. Shanks, K. J. Holyoak, \& D. L. Medin (Eds.), The psychology of learning and motivation: Vol. 34. Causal learning (pp. 47-88). San Diego: Academic Press.

Waldmann, M. R. (2000). Competition among causes but not effects in predictive and diagnostic learning. Journal of Experimental Psychology: Learning, Memory, \& Cognition, 26, 53-76.

WALDMANN, M. R. (2001). Predictive versus diagnostic causal learning: Evidence from an overshadowing paradigm. Psychonomic Bulletin \& Review, 8, 600-608.

WALDMAnN, M. R., \& HaGmaYer, Y. (1999). How categories shape causality. In M. Hahn \& S. C. Stoness (Eds.), Proceedings of the
Twenty-First Annual Conference of the Cognitive Science Society (pp. 761-766). Mahwah, NJ: Erlbaum.

Waldmann, M. R., \& Holyoak, K. J. (1992). Predictive and diagnostic learning within causal models: Asymmetries in cue competition. Journal of Experimental Psychology: General, 121, 222-236.

Wasserman, E. A., Elek, S. M., Chatlosh, D. L., \& BaKer, A. G. (1993). Rating causal relations: Role of probability in judgments of response-outcome contingency. Journal of Experimental Psychology: Learning, Memory, \& Cognition, 19, 174-188.

WAsserman, E. A., \& Neunaber, D. J. (1986). College students' responding to and rating of contingency relations: The role of temporal contiguity. Journal of the Experimental Analysis of Behavior, 46, 15-35.

White, P. A. (2003). Making causal judgments from the proportion of confirming instances: The pCI rule. Journal of Experimental Psychology: Learning, Memory, \& Cognition, 29, 710-727.

Young, M. E. (1995). On the origin of personal causal theories. Psychonomic Bulletin \& Review, 2, 83-104.

\section{NOTES}

1. A common view in the literature is that "perceptual causality" (e.g., Michotte, 1946/1963) is fundamentally different from other forms of causal learning, and does not draw on any inferential components (see Scholl \& Tremoulet, 2000, for an overview). However, it is quite plausible that perceptual causality, rather than being modular, shares inferential components common to causal learning in general (see Cheng, 1993, for an inferential analysis of the launching effect) and adapts them to the constraints of the specific percept (Scholl \& Nakayama, 2002).

2. Of course there are many examples (to be discussed later on) where contiguity is violated, for example in the relation between drinking hemlock and death a few hours later. Hume's argument was that causation at a distance — spatial or temporal — does not exist, even though the appearance to our senses may sometimes deceive us into believing otherwise. In the hemlock example, for instance, the cause "drinking hemlock" sets in motion a causal chain of many events, each of which is caused by its predecessor and in turn produces the next one. Thus, although cause and (final) effect are not contiguous, the links in the chain are with respect to each other.

3 . The problem with allocating events to the four cells of the contingency matrix has been raised already by Hammond and Paynter (1983). Their analysis was concerned with animal conditioning, and their suggested solution - to employ very small units of temporal analysis (i.e., trials) combined with weighting formulas "that categorize the conjointness of events in a quantitative fashion, on the basis of temporal order and proximity effects that have been demonstrated through experimentation" (p. 546) - has only limited use for the study of human learning. This is because small units of analysis might yield a descriptive fit (given the appropriate degrees of freedom), but lack psychological plausibility, as will become evident later on. 